\title{
Diode Pumped Bi-doped Fiber Laser Operating at 1360nm
}

\author{
N. K. Thipparapu,* A. A. Umnikov, S. Jain, P. Barua, and J. K. Sahu \\ Optoelectronics Research Centre, University of Southampton, Highfield, Southampton, SO17 1BJ, U.K. \\ *Corresponding author e-mail address: nkt1n12@soton.ac.uk
}

\begin{abstract}
Bi-doped phosphosilicate fibers are fabricated by MCVD-solution doping technique under different oxidation conditions. Fibers are evaluated for unsaturable loss and laser performance. A $22 \mathrm{~mW}$ all-fiber Bi-laser is demonstrated at $1360 \mathrm{~nm}$ by LD pumping at $1267 \mathrm{~nm}$. OCIS codes: (060.2280) Fiber design and fabrication; (060.2270) Fiber characterization; (060.3510) Lasers, fiber;
\end{abstract}

\section{Introduction}

Exploring fiber lasers and amplifiers in new wavelength bands have been the research interest for decades. The $1150-1500 \mathrm{~nm}$ wavelength region is one of such bands with demanding applications such as wideband optical fiber communication, medicine, spectroscopy, and astronomy. Bismuth (Bi) doped fibers have paved the way to develop lasers and amplifiers in this wavelength band, thanks to its broad luminescence characteristics. Bi-doped aluminosilicate, phosphosilicate and germanosilicate fibers have shown luminescence around $1150 \mathrm{~nm}, 1300 \mathrm{~nm}$ and $1450 \mathrm{~nm}$ respectively $[1,2]$. In particular, Bi-doped fibers which exhibit luminescence around 1300nm has specific importance as their operating wavelength region coincide with the second telecommunication window. Bi-doped phosphogermanosilicate fibers have been used to demonstrate lasers from 1280-1360nm with a 30W Raman fiber laser operating at $1230 \mathrm{~nm}$ as a pump [3]. Among these, the reported efficiency of laser operating at 1360nm was $9.5 \%$ and $\sim 18 \%$ at low and high pump powers, respectively. Moreover a $100 \mathrm{~m}$ long Bi-doped fiber was used for laser demonstration. In this paper, we report the fabrication of Bi-doped phosphosilicate fibers (BPSFs) using MCVD-solution doping technique under different atmospheric conditions. The fibers have been characterized for their unsaturable loss (UL) at different pump wavelengths. An all-fiber Bi-doped laser in a ring cavity has been demonstrated by direct diode pumping at $1267 \mathrm{~nm}$ with a laser output power of $22 \mathrm{~mW}$ operating at a wavelength of $1360 \mathrm{~nm}$. The fiber length used in our experiment was less than $50 \mathrm{~m}$.

\section{Fiber fabrication and characterization}

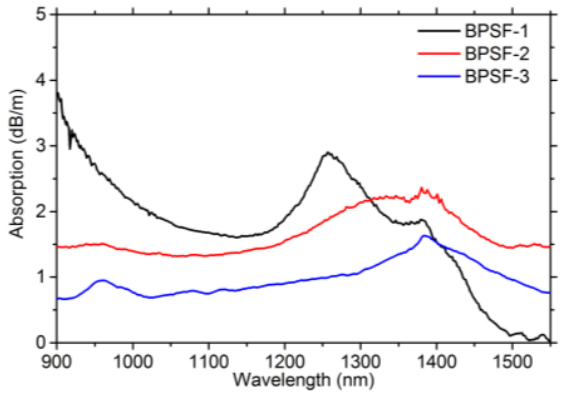

Table 1. UL of different BPSFs measured at two pump wavelengths

\begin{tabular}{c|c|c|c|c}
\hline \multicolumn{2}{c|}{ BPSF fabricated } & BPSF-1 & BPSF-2 & BPSF-3 \\
\hline $\begin{array}{c}\text { Preform fabrication condition during } \\
\text { sintering and collapsing stages } \\
\text { ( flow of } \mathrm{O}_{2} \text { to He in SCCM) }\end{array}$ & $250: 250$ & $400: 100$ & $500: 0$ \\
\hline \multirow{2}{*}{$1210 \mathrm{~nm}$ pump } & $\mathrm{UL}(\mathrm{dB} / \mathrm{m})$ & 0.96 & 0.3 & 0.14 \\
\cline { 2 - 5 } & \%UL & 46 & 17 & 13.5 \\
\hline \multirow{2}{*}{$1267 \mathrm{~nm}$ pump } & $\mathrm{UL}(\mathrm{dB} / \mathrm{m})$ & 0.61 & 0.22 & 0.08 \\
\cline { 2 - 5 } & \%UL & 24.5 & 11 & 7.2 \\
\hline
\end{tabular}

Fig. 1. Absorption spectra of Bi-doped phosphosilicate fibers

Bi-doped preforms have been fabricated by depositing phosphosilicate soot under similar fabrication conditions. The soot body was impregnated by the same Bi solution for all the preforms. The only difference between fabricated preforms was the variation of Oxygen $\left(\mathrm{O}_{2}\right)$ and Helium $(\mathrm{He})$ flow ratio during the porous layer sintering and the preform collapse stages, while maintaining the total gas flow as constant (see Table 1). The preforms were drawn into fibers (from here onwards named BPSF-1, BPSF-2 and BPSF-3) with a core and clad diameter of $13 \mu \mathrm{m}$ and $100 \mu \mathrm{m}$, respectively. The index difference $(\Delta \mathrm{n})$ between the core and clad was found to be 0.004 for all BPSFs. The absorption spectra of all BPSFs were measured by cut-back method using a white light source (WLS) and an optical spectrum analyzer (OSA) and are shown in Fig. 1. The influence of fabrication conditions on absorption characteristics can be seen from Fig. 1. BPSF-1 which was fabricated with 1:1 of $\mathrm{O}_{2}$ to He ratio has shown an absorption peak around $1255 \mathrm{~nm}$ accompanying a small water peak at $1380 \mathrm{~nm}$. Whereas in BPSF-2, fabricated under excess $\mathrm{O}_{2}$ (4: 1 ratio of $\mathrm{O}_{2}$ to $\mathrm{He}$ ), the $1255 \mathrm{~nm}$ peak shifted to around $1335 \mathrm{~nm}$ and it was less pronounced. In BPSF3 , that was fabricated under complete $\mathrm{O}_{2}$ atmosphere the $1255 \mathrm{~nm}$ peak disappeared. Also, the absorption in BPSF-3 reduced compared to BPSF-1 and BPSF-2. The UL was measured for all BPSFs at two wavelengths 1210 and $1267 \mathrm{~nm}$ (see Table 1) using an established method [4]. The UL was around $24.5 \%(46 \%), 11 \%(17 \%)$ and $7.2 \%$ $(13.5 \%)$ at pump wavelength $1267 \mathrm{~nm}(1210 \mathrm{~nm})$ in BPSF-1, BPSF-2 and BPSF-3, respectively. This indicated that the $\mathrm{O}_{2}$ rich atmospheric condition during preform fabrication reduces the UL in BPSFs. 


\section{Bi-doped fiber laser: Experimental set up and Results}
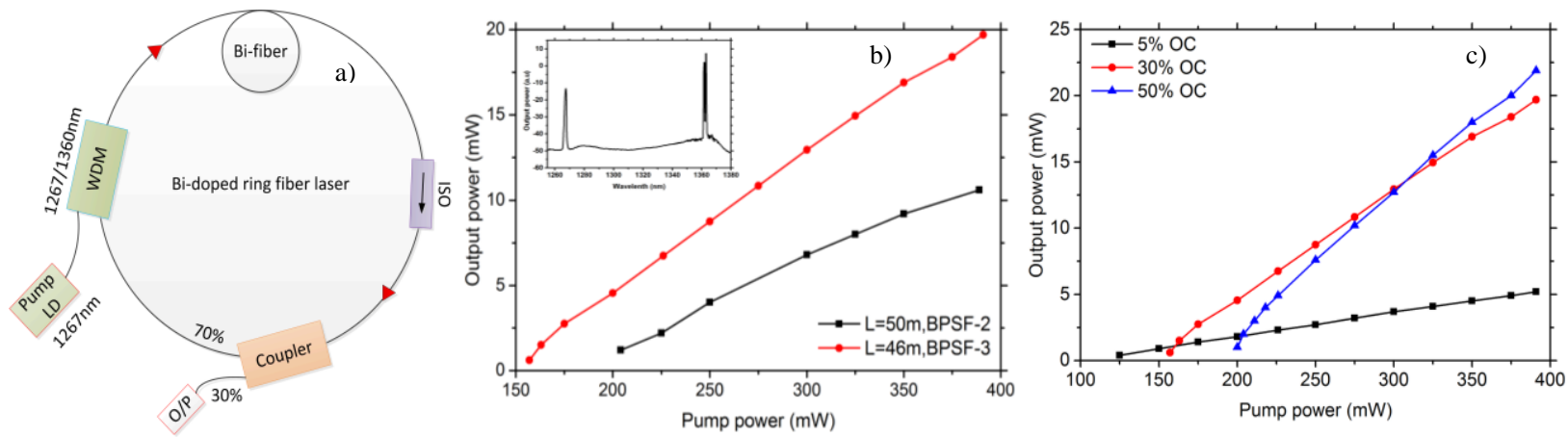

Fig. 2. a) Schematic experimental set up of Bi-doped fiber laser, b) Output power vs launched pump power for optimum lengths of BPSF-2, BPSF-3 (Inset shows the laser spectrum at $1360 \mathrm{~nm}$ of BPSF-3), c) Output power vs launched pump power with different output coupling (OC) for 46m long BPSF-3.

The experimental set up of Bi-doped fiber laser in a ring cavity is shown in Fig. 2 (a). It consists of a fiber pigtailed pump LD operating at $1267 \mathrm{~nm}$, a 1267/1360nm wavelength division multiplexer (WDM), an Isolator, and an optical coupler for cavity feedback. An additional WDM was used to separate pump from signal in the output port of the coupler. The maximum available pump power from the LD is $390 \mathrm{~mW}$. The $1267 \mathrm{~nm}$ pumping wavelength was chosen due to lower UL in BPSF among the available pump LDs. The absorption values at $1267 \mathrm{~nm}$ pump wavelength measured by WLS were found to be $2.8 \mathrm{~dB} / \mathrm{m}, 1.95 \mathrm{~dB} / \mathrm{m}$ and $1 \mathrm{~dB} / \mathrm{m}$ in BPSF-1, BPSF-2 and BPSF-3, respectively. At first, a coupler with an input/output coupling ratio of 70/30 was used for BPSF-1. No lasing action was obtained in this fiber at any fiber length despite being highest absorption at the pump wavelength. Next, BPSF-2 was tested using the same coupling ratio and it shows lasing at a wavelength of $1360 \mathrm{~nm}$. The length of the fiber was optimized to achieve maximum laser efficiency. The optimum fiber length was found to be $50 \mathrm{~m}$ with laser efficiency of $5 \%$ and the maximum output power was $10 \mathrm{~mW}$ as shown in Fig. 2 (b). Subsequently, BPSF-3 was tested which exhibited a laser efficiency of $8 \%$ with an output power of $20 \mathrm{~mW}$ for a fiber length of $46 \mathrm{~m}$. The threshold pump power was $157 \mathrm{~mW}$. The laser spectrum of BPSF-3 taken by an OSA is shown in the inset of Fig. 2 (b). We then varied the output coupling ratio in the ring cavity. The laser efficiency in BPSF-3 was increased to $11 \%$ with an output power of $22 \mathrm{~mW}$ for the 50/50 coupling ratio as shown in Fig. 2 (c). The performance of BPSF laser can be improved further by optimizing the preform fabrication conditions. From this study, it is evident that an absorption peak around $1255 \mathrm{~nm}$ leads to higher UL and thereby impairing the laser performance.

\section{Conclusions}

In conclusion, we fabricated BPSFs under different oxidation conditions by standard MCVD-solution doping technique. Absorption, UL and laser efficiency have been measured in all BPSFs and found to be strongly correlated with the fabrication conditions. The UL was found to be low at $1267 \mathrm{~nm}$ in comparison with $1210 \mathrm{~nm}$ pump wavelength. A Bi-doped fiber laser operating at $1360 \mathrm{~nm}$ has been demonstrated with a laser efficiency of $11 \%$ by direct diode pumping at $1267 \mathrm{~nm}$.

\section{Acknowledgement}

This work was supported by the UK Engineering and Physical Sciences Research Council grant EP/I01196X/1 (Hyperhighway).

\section{References}

[1]I. A. Bufetov, M. A. Melkumov, S. V. Firstov, K. E. Riumkin, A. V. Shubin, V. F. Khopin, A. N. Guryanov, and E. M. Dianov, "Bi-doped optical fibers and fiber lasers," IEEE J. Sel. Topics Quantum Electron. 20, 111-125 (2014).

[2]N. K. Thipparapu, S. Jain, A. A. Umnikov, P. Barua, and J. K. Sahu, “1120 nm diode-pumped Bi-doped fiber amplifier,” Opt. Lett. 40 , 2441 2444 (2015).

[3] I. A. Bufetov, M. A. Melkumov, V. F. Khopin, S. V. Firstov, A. V. Shubin, O. I. Medvedkov, A. N. Guryanov, and E. M. Dianov, "Efficient Bi-doped fiber lasers and amplifiers for the spectral region 1300-1500 nm," in Proc. SPIE LASE, 7580 (International Society for Optics and Photonics), 758014-758014 (2010).

[4]M. P. Kalita, S. Yoo, and J. Sahu, "Bismuth doped fiber laser and study of unsaturable loss and pump induced absorption in laser performance,” Opt. Express 16, 21032-21038 (2008). 